\title{
Executive control network connectivity strength protects against relapse to cocaine use
}

\author{
Meredith J. McHugh ${ }^{1,2}$, Hong Gu${ }^{1}$, Yihong Yang ${ }^{1}$, Bryon Adinoff ${ }^{3,4}$, Elliot A. Stein ${ }^{1}$
}

1. Neuroimaging Research Branch, National Institute on Drug Abuse-Intramural Research Program, Baltimore, MD

2. Orygen, The National Centre for Excellence in Youth Mental Health, Melbourne, Australia.

3. Department of Psychiatry, UT Southwestern Medical Center, Dallas, TX

4. VA North Texas Health Care System, Dallas, TX

\section{Corresponding author:}

Meredith J. McHugh, Orygen, The National Centre of Excellence in Youth Mental Health, 35 Poplar Rd, Melbourne, VIC, Australia. Ph. +61 93422993 Email: meredith.mchugh@orygen.org.au

Keywords: cocaine, relapse, addiction, executive control, resting state connectivity

Abstract word length: 250 words

Body text word length: 4978 words

Number of Tables: 3 
Number of Figures: 4

Supplementary materials: 1 Figure, Supplementary Methods)

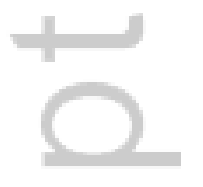

\section{Abstract}

Cocaine addiction is characterized by notoriously high relapse rates following treatment. Recent efforts to address poor treatment outcomes have turned to potential neural markers of relapse risk. Accordingly, the present study examined resting state functional connectivity (rsFC) within and between three large-scale cortical networks: the Default Mode Network (DMN), Salience Network (SN) and Executive Control Network (ECN). All three have been implicated in relapse-related phenomena including craving, withdrawal and executive control deficits. Forty-five cocaine-dependent individuals and 22 healthy controls completed 6-min resting fMRI scans, The Wisconsin Card Sorting Task, Continuous Performance Test and Cocaine Craving Questionnaire. Cocaine-dependent individuals completed all measures in the final week of a residential treatment episode. Ten control and 9 abstinent cocaine-dependent individuals returned for 3-6 month follow-up scan visits. A group-level Independent Component Analysis was employed to generate ECN, DMN and SN components. For individuals abstinent up to day 30 post-treatment $(n=21)$ we found enhanced pre-discharge rsFC between the left ECN and right ECN and SN as well as between the right ECN and left ECN. Left ECN rsFC effects remained elevated 3-6 months later among abstinent cocaine-dependent individuals. Relapse was related to fewer years of education and more years smoking but no other demographic, clinical, treatment and neurocognitive characteristics. Findings suggest that interhemispheric ECN and ECN-SN connectivity strength may protect against relapse to cocaine use following treatment. These patterns of enhanced 
interhemispheric network connectivity may reflect a greater capacity to engage executive control processes when faced with opportunities to use cocaine post-treatment.

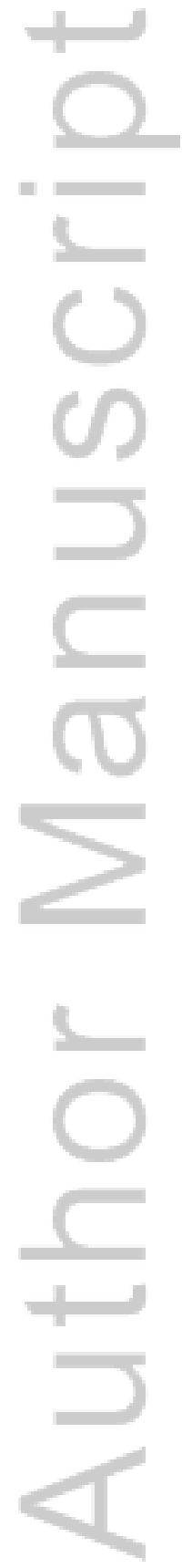




\section{Introduction}

Cocaine addiction is a chronic, relapsing neuropsychiatric disease (Dutra et al. 2008). Current understanding of factors driving relapse to cocaine use derives primarily from clinical observations (Poling, Kosten, and Sofuoglu 2007) and preclinical studies demonstrating that reactivity to stress (Erb 2009), drug craving (See 2005), and deficits in prefrontal "'executive"' control (Chen et al. 2013) drive the reinstatement of extinguished cocaine-seeking (Kalivas, 2008). Despite this understanding, relapse rates remain high, with nationwide surveys of outpatient cocaine treatment mean relapse rates approaching 70\% (Dutra et al. 2008).

Recent research efforts to address high relapse rates have focused on neural markers of relapse risk (Marhe et al. 2013; McHugh et al. 2014; McHugh et al. 2013; Adinoff et al. 2015). Such efforts have occurred alongside an explosion of interest in large-scale cortical networks and evidence that intrinsic fluctuation in the ongoing resting activity within these networks demonstrate alterations in such neuropsychiatric conditions as depression, schizophrenia (Greicius 2008) and addiction (Fedota \& Stein, 2015; Sutherland, McHugh, Pariyadath, \& Stein, 2012). Three such networks, the default mode network (DMN), executive control network (ECN) and salience network (SN), have been consistently linked to relapse-relevant phenomena, including drug craving, withdrawal from drugs of abuse and deficits in executive control (for reviews see Fedota and Stein, 2015; Naqvi and Bechara, 2010; Sutherland et al., 2012).

The DMN (primary hubs in the medial prefrontal and posterior cingulate cortices) mediates internally oriented, self-referential processing (Buckner, Andrews-Hanna, and Schacter 2008), such as rumination on drug use (Sutherland et al. 2012). DMN engagement is also typically accompanied by ECN (typically lateral prefrontal, parietal, and middle temporal cortices) disengagement and coincident deficits 
in cognitive and attentional processes mediated by this network. For example, in dependent tobacco smokers, Lerman and colleagues showed that acute withdrawal from nicotine produces a shift in ongoing resting state activity towards greater coupling between SN and DMN and less SN-ECN engagement along with greater self-reported craving and poorer capacity to engage ECN regions during a subsequent working memory task (Lerman et al. 2014). More generally, a hallmark of cocaine dependence is a pattern of functional and structural alterations within the ECN (particularly frontal cortical regions) and associated deficits in executive control which are, in turn, implicated in the maintenance of, and return to cocaine-use following treatment (Goldstein and Volkow 2002).

Finally, cortical regions which together form the SN (i.e., dorsal anterior cingulate and anterior insula cortices) consistently activate during the presentation of drug-associated cues (Naqvi and Bechara 2010). The putative function of the SN is to detect internally or externally generated salient events (e.g., drug-associated cues) and direct the allocation of attentional and cognitive resources in response to each event (Menon and Uddin 2010). A current view is that the SN may achieve this by orchestrating toggling between ECN and DMN, engaging whichever network is needed to best generate an adaptive response (Menon and Uddin 2010).

Based on the aforementioned observations, several hypotheses can be derived regarding the likely contribution of intrinsic connectivity within and between these three networks to relapse susceptibility. Firstly, if the DMN supports drug craving and rumination on drug use, we would expect greater intrinsic connectivity within the DMN among individuals at greatest risk of relapse. Secondly, if the SN enhances which of the ECN and DMN is more readily engaged, we would expect greater intrinsic connectivity between the SN and DMN and less connectivity between the SN and ECN to predict relapse susceptibility. Thirdly, given that intrinsic fluctuations within resting state networks reflect both current 
state and capacity to engage networks during tasks (e.g., Lerman et al., 2014), reduced intrinsic connectivity within the ECN should reflect a reduced capacity to engage executive control processes when faced with opportunities to use cocaine post-treatment, and hence greater relapse susceptibility. In addition we expected the hypothesized patterns of intrinsic connectivity to be associated with deficits in executive control and sustained attention. Here we focused on perseverative responding on the Wisconsin Card Sorting Task (WCST; Heaton 1993) based on evidence that cocaine-dependent individuals exhibit deficits on this measure whereas other cognitive functions seem preserved (Woicik et al. 2011). We also examine performance on the Continuous Performance Task (CPT; Connor and Staff 2004), which assesses both inhibitory control and sustained attention, processes impacted by abstinence from drugs of abuse (Powell et al. 2004; Dalley, Everitt, and Robbins 2011; Tomasi et al. 2010; Goldstein et al. 2004), and considered central to long-term recovery in addiction (Rezapour et al. 2016).

In the present study, we tested the aforementioned hypotheses in a group of cocaine-dependent individuals using resting state functional connectivity ( $\mathrm{rsFC}$ ) measured during the final week of a residential treatment episode. Resting connectivity data from the present sample have been reported in two previous studies examining relapse risk as a function of hypothesized striatal (McHugh et al. 2013) and amygdala (McHugh et al. 2014) circuits and one study examining resting connectivity within circuits that might drive relapse-related differences in basal neural activity (resting Cerebral Blood Flow, rCBF; Adinoff et al. 2015). Relapse to drug use is a complex neuropsychiatric phenomena involving multiple neurobiological mechanisms (Stewart 2008b). Markers of relapse risk, and targets for treatment are thus likely to span multiple neurobiological systems. In the present study we move away from discrete circuits derived from a priori seed regions (as in our two previous studies, McHugh et al., 2013, 2014) and 
instead employ a data-driven Independent Component Analyses (ICA) approach to study large-scale cortical networks (DMN, ECN, SN) previously shown to be involved in relapse-related phenomena.

\section{Methods and Materials}

\section{Participants.}

Forty-five treatment-seeking individuals meeting DSM-IV criteria for cocaine-dependence (CD) were recruited from three residential cocaine-addiction treatment facilities. An additional 21 healthy control (HC) individuals with no history of drug abuse or dependence were recruited in parallel from the general community. All study participants had no history of major illness, an estimated IQ of 70 or above [per the Wechsler test of adult reading (WTAR)], were right-handed, did not meet criteria for any neurological or active axis I disorder (other than substance use disorders) and were not currently on psychotropic medications. Other drug use among cocaine-dependent subjects was not a condition for exclusion as long as cocaine dependence was the primary diagnosis.

All aspects of the research protocol were reviewed and approved by the Institutional Review Boards of the University of Texas Southwestern Medical Center at Dallas and the Veterans Administration North Texas Health Care System. Subjects provided informed consent prior to study participation.

Measures of executive control, sustained attention and craving

- Conners' Continuous Performance Test II (CPT-II). The CPT-II measures sustained attention and inhibitory control (Connor and Staff 2004). Respondents are required to press the space bar when any letter except the target letter " $X$ " appears. In the present study, attention was indexed with errors of 
omission (not pressing for letters other than $\mathrm{X}$ ), reaction time and standard error of reaction time. Errors of commission (failing to inhibit response to $\mathrm{X}$ ) index both attention and inhibitory control.

Wisconsin Card Sorting Task. The Wisconsin Card Sorting Test (WCST) (Heaton 1993) assesses set shifting. Participants are asked to sort 128 cards (two sets of 64) according to one of three principles: color, form, or number of symbols displayed on each card. The principal shifts every 10 cards and participants must notice and learn to sort the cards according to the new principle. Here we focused on perseverative responding, the failure to change strategies following a principle shift.. The total number of perseverative responses was natural log transformed to normalize the distribution prior to conducting group contrasts.

Cocaine Craving Questionnaire (CCQ). The Cocaine Craving Questionnaire (CCQ-Brief) (Tiffany et al. 1993) is a 10-item self-report measure adapted from the original 45-item CCQ-Now that asks subjects to indicate how much they agree or disagree with statements related to cocaine craving. Items are statements relating to an individual's current state of cocaine craving which they rate on a 7point likert scale ranging from 1 (strongly disagree) to 7 (strongly agree). Thus scores on this measure can range from 10 to 70 .

Procedure

CD participants were admitted to one of three residential treatment facilities as soon as possible following their last cocaine use. All three programs followed the Minnesota Model for the treatment of cocaine-addiction (Cook 1988). Demographic information, drug use history, and IQ was typically collected during the first week of treatment. Abstinence was verified by urine drug screens collected throughout treatment. During their final week of treatment, CD participants completed a 5-min high resolution structural scan and a 6-min resting fMRI scan during which they were instructed to lie as still 
as possible with their eyes open. The WCST, CPT and CCQ measures were completed by HC and CD participants as part of a larger neurocognitive assessment completed on a separate day during the same week as the scan session.

Following discharge from treatment CD participants completed two follow-up sessions each week (once over the phone) for up to 26 weeks. Each session included a structured interview assessing substance use since their previous session, and a urine drug screen for in-person sessions. Relapse was defined as any use of cocaine or amphetamine post-discharge. Date of relapse was scored as the day of first use, or the day of their first missed appointment if participants missed two consecutive appointments. Participants who relapsed were discharged from the study and excluded from further follow-up. At three to six months following the pre-discharge scan session, a subgroup of HC participants $(n=10)$ and CD participants who remained abstinent $(n=9)$ completed follow-up scan sessions along with neurocognitive assessments. Procedures were identical to those completed pre-discharge.

\section{MRI Data Acquisition}

Thirty-six 3mm thick functional MRI slices were acquired on a 3-T Phillips MR scanner with an eight channel RF coil. Slices were obtained in the axial plane parallel to the AC-PC line using single-shot gradient-echo echo-planar imaging (EPI) sequence with a matrix of $64 \times 64$, echo time (TE) of $25 \mathrm{ms,}$ repetition time (TR) of $1.7 \mathrm{~s}$, flip angle (FA) of $70^{\circ}$, field of view (FOV) of $208 \mathrm{~mm} \times 208 \mathrm{~mm}$. This yielded an in-plane resolution of $3.25 \mathrm{~mm} \times 3.25 \mathrm{~mm}$. High resolution anatomical T1 images were acquired using 3D magnetization prepared rapid gradient-echo (MPRAGE) sequence with a TR/TE $8.2 / 3.8 \mathrm{~ms}, \mathrm{FA}$ of $12^{\circ}$, and voxel size of $1 \mathrm{~mm} \times 1 \mathrm{~mm} \times 1 \mathrm{~mm}$.

\section{Data processing}


Data were preprocessed and analyzed using AFNI (Cox 1996), FreeSurfer (http://surfer.nmr.mgh.harvard.edu/) and SPSS version 20. Following image reconstruction, resting data were submitted to slice-timing correction, motion correction, and quadratic detrending of time series data. A band-pass temporal filter was applied to restrict signal variation to frequencies between 0.01 and $0.1 \mathrm{~Hz}$ (Biswal et al. 1995). Resting data images were then registered to standard (Talairach) space with a resampled resolution of $3 \mathrm{~mm} \times 3 \mathrm{~mm} \times 3 \mathrm{~mm}$ and smoothed with an isotropic $6 \mathrm{~mm}$ full-width halfmaximum (FWHM) Gaussian kernel. An unbiased groupwise non-linear registration method (Geng et al. 2009) was used to improve cross-subject alignment. Additional procedures for controlling head motion in the present study are provided in the Supplementary methods section.

To generate group-level component maps for the DMN, ECN and SN, we employed a subjectorder-independent group ICA (gICA; Zhang et al. 2010) using the FSL MELODIC algorithm (http://fsl.fmrib.ox.ac.uk/fsl/fslwiki/MELODIC). A 24 component solution produced the best spatial match (established via visual inspection) for the canonical networks of interest (Shirer et al. 2012). This procedure was then repeated a further nine times, each time randomizing the subject order prior to a cross-subject concatenation. The output of these 10 group ICAs was then submitted to a single meta ICA (Zhang et al. 2010) to produce a final set of subject-order-independent group-level component maps.

A dual-regression procedure (Filippini et al. 2009) was employed to generate individual-level connectivity maps for each component of interest, controlling for fluctuations in BOLD signals from cerebrospinal fluid and white matter and six motion parameters by modelling these as nuisance variables (see Supplementary Methods for further details).

\section{Data Analysis}


To examine the relationship between pre-discharge network connectivity and early relapse risk, CD individuals were split into two groups (Relapse and Nonrelapse) based on relapse status at day 30 post-discharge, a timeframe consistent with the DSM-IV criteria for early remission from cocainedependence (American Psychiatric Association 1994). Because recruitment for this study was conducted on the basis of DSM-IV criteria for cocaine dependence, we have maintained the DSM-IV criteria for early remission to remain consistent. Demographic, clinical and neurocognitive characteristics as well as motion during the scan were compared between Relapse, Nonrelapse and HC individuals with betweengroup ANOVAs, post-hoc $t$-tests and Chi-squared tests (for differences in frequencies).

Voxelwise relapse-related differences in network connectivity were assessed for each network of interest using a general linear mixed-effects model with group (Relapse vs Nonrelapse) and years of education (which significantly differed between the two CD groups) as fixed factors. Because we were specifically interested in between and within network connectivity, we created a compound mask of the three networks derived from the mean subject-level network connectivity maps (voxelwise $p_{\text {uncorrected }}=$ $.0001)$ including only voxels that contributed to at least one network of interest. This mask was applied for all voxelwise analyses reported in this paper. For relapse-related contrasts, an uncorrected voxelwise threshold was set at $p=.005$, with effects reported if they first passed a clusterwise threshold of $p=.05$ (based on Monte Carlo simulations using the AlphaSim program in AFNI). Where significant relapserelated differences were present, post hoc voxelwise contrasts were conducted between the HC and CD groups. As the focus of the present paper is identifying patterns of network connectivity which predicted relapse following treatment, we did not conduct a priori contrasts between $\mathrm{HC}$ and CD groups. The sole purpose of these post-hoc contrasts was to facilitate interpretation of relapse-related group difference (i.e., which group is more like the $\mathrm{HC}$ group). 
To determine whether pre-discharge differences related to early relapse sustain (or elevate) at 3-6 months post-discharge we extracted average connectivity within clusters where significant 30-day relapse-related differences were present pre-discharge. Please note that "pre-discharge" here refers to each participants initial scan session, which for CD participants was conducted during the final week of treatment. We then conducted within-subject (Nonrelapse pre-discharge vs 3-6 months) and betweensubject (Nonrelapse at 3-6 months vs Relapse at pre-discharge, with years of education included as a covariate) contrasts on average connectivity within each cluster. Contrasts were Bonferroni-corrected for multiple comparisons. As a comparison of stability in resting connectivity across time we also assessed pre-discharge vs 3-6 month connectivity in HC participants. Bayes Factors (BF) were computed for each within-subject contrast (Rouder et al. 2009). The BF provides a likelihood ratio in favor of the null or alternative hypothesis. That is, where mean differences are not significant, the likelihood of drawing this mean difference from a null distribution relative to an alternative distribution where mean differences are truly greater than zero.

Finally, for network components where relapse-related differences were present, we examined the relationship between component connectivity and neurocognitive performance. Specifically, in separate analyses we searched, voxel-wise, for regions where network component connectivity varied as a function of natural log transformed perseverative responding on the WCST and commission errors on the CPT. To minimize the number of post-hoc contrasts we focus on the CPT commission errors (rather than other indices derived from this measure) as taps into both impulse control and sustained attention, processes impacted by abstinence from drugs of abuse (Powell et al. 2004; Dalley, Everitt, and Robbins 2011; Tomasi et al. 2010; Goldstein et al. 2004). We did not examine craving (CCQ) scores in this manner because $38 \%$ of the $\mathrm{CD}$ group reported no craving resulting in a highly skewed distribution. Effects were 
considered significant if they passed an uncorrected voxelwise $p_{\text {uncorrected }}=.005$ threshold and a corrected clusterwise threshold of $p_{\text {corrected }}=.05$.

\section{Results}

Relapse by day 30, 3 months and 6 months

As illustrated in Table 1, 24 of 45 CD individuals relapsed to cocaine use and one CD individual to amphetamine use within the first 30 days post-discharge from treatment. Of the 21 Nonrelapse individuals, 12 remained abstinent at 3 months, and of these 8 were still abstinent at 6 months.

Demographic, clinical and neurocognitive characteristics

Table 1 illustrates demographic and clinical characteristics, head motion during the resting scan, as well as neurocognitive and treatment characteristics of Relapse, Nonrelapse and HC individuals.

Relapse by day 30 post-discharge was associated with fewer years of education $(p=.01)$ and more years smoking $(p=.05)$ relative to Nonrelapse participants. No other demographic, clinical or neurocognitive characteristics (all $p s>.10$ ) were associated with relapse by day 30. Relapse, Nonrelapse and HC participants did not differ in head motion during the pre-discharge scan $(p=.62)$. Table 1 also outlines demographic, neurocognitive and drug use characteristics of the HC participants and indicates where they differed significantly from either CD group.

Early relapse and pre-discharge network connectivity 
Fig S1 displays group component maps for the DMN, ECN (left and right ECN components) and SN. Differences in resting connectivity between Relapse and Nonrelapse participants were seen for left ECN and right ECN components only. As illustrated in Table 2 and Fig 1, relative to Relapse individuals, Nonrelapse individuals evidenced heightened connectivity between the left ECN and dorsolateral prefrontal portions of the right ECN (right middle and superior frontal gyrus Figs 1A and 1C). They also evidenced enhanced connectivity between the left ECN and portions of the SN component (right dorsal ACC and left cerebellum, Fig 1B and Table 2). For the right ECN Nonrelapse individuals evidenced enhanced connectivity with a left middle temporal gyrus (MTG) portion of the left ECN, relative to those who relapsed (Fig 2, Table S1). Post-hoc contrasts between HC and Nonrelapse and HC and Relapse individuals did not reveal significant differences in right or left ECN connectivity within any of the aforementioned regions. Finally, we conducted the Relapse vs Nonrelapse contrasts with and without years of smoking included as a covariate. The inclusion of years of smoking had no impact on the significance of effects observed and reported here.

\section{Cocaine abstinence and network connectivity at 3-6 months follow-up}

Nine of the 12 individuals abstinent for 3-6 months returned for a follow-up scan and neurocognitive assessment. Eight individuals completed this follow-up assessment at 3 months and one at or 6 months post-discharge. Of the $21 \mathrm{HC}$ individuals who completed pre-discharge scans, 10 returned for a 3 month $(n=8)$ or 6 month $(n=2)$ follow-up scan.

To determine whether pre-discharge connectivity effects are still present among individuals who remain abstinent at 3-6 months follow-up $(n=9)$, we extracted average connectivity at both pre-discharge and follow-up in relapse-related clusters (see Figure 1 and 2). We then compared average connectivity 
within these clusters between Nonrelapse individuals at follow-up and Relapse individuals pre-discharge $(n=24)$. As illustrated in Fig 3, individuals abstinent at day 30 continued to exhibit significantly enhanced connectivity in the right $\operatorname{MFG}(F[30,1]=7.221, p=.012)$ and right $\mathrm{dACC}(F[1,30]=8.090, p=$ .008) 3-6 months post-treatment for the left ECN, relative to pre-discharge connectivity among individuals who relapsed within 30 days $(n=24)$. Within-subject contrasts demonstrated that connectivity within each of these clusters in the 3-6 month abstinent group did not significantly differ from predischarge (left ECN-right MFG: $t$ [9] = .144, $p=.91$; left ECN-right dACC: $(\mathrm{t}[9]=.127, p=.90)$. Bayes Factors calculated for each contrast favored the null hypothesis (left ECN-right MFG contrast, BF = 2.49; left ECN-right dACC contrast, $\mathrm{BF}=1.23$ ). This provides some evidence that these putatively protective effects were sustained and did not significantly enhance or decline with longer-term abstinence from cocaine use, though Kass and Raftery (1995) cautions that the Bayes Factors reported here provide only weak evidence to reject the null hypotheses of no change. Similarly, among HC participants who returned for follow-up scans $(n=10)$, no differences were observed between pre-discharge and 3-6 months resting connectivity in these clusters (all $p \mathrm{~s}>.476 ; \mathrm{BF}=1.02$ and 1.70 in favor of the null), suggesting a similar stability.

\section{Network connectivity and neurocognitive performance.}

For the two networks where relapse-related effects emerged (left and right ECN), we searched, voxelwise, for regions where network component connectivity varied as a function of neurocognitive performance. As illustrated in Fig 4 and Table 3, less perseverative responding on the WCST was associated with enhanced connectivity between the left ECN and right ECN, specifically in a right MFG region adjacent to that associated with relapse-related connectivity differences. For the right ECN, less perseverative responding was associated with enhanced within- network connectivity, specifically greater resting 
connectivity strength with the right supramarginal gyrus and right middle temporal gyrus (see Table 3). Commission errors on the CPT were not significantly related to voxelwise connectivity of either the left or right ECN.

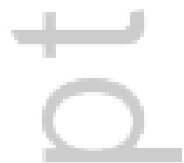

\section{Discussion}

Predicting and preventing relapse to cocaine use remains a primary challenge in efforts to improve poor treatment outcomes for cocaine-dependent individuals. Here we show that intrinsic connectivity within and between large-scale cortical networks implicated in craving and executive control could serve as markers of relapse risk and thus potential treatment targets. Firstly, we observed a pattern of enhanced interhemispheric connectivity within prefrontal components of the ECN among those individuals who maintained abstinence from cocaine use for up to 30 days post-discharge from residential treatment. Interestingly, this pattern of elevated frontal interhemispheric connectivity seen during the final week of treatment was still present 3-6 months post-treatment among the subgroup of individuals still abstinent at this later time point. Together these findings suggest that frontal interhemispheric connectivity may confer a trait-like protective effect, perhaps enabling CD individuals to more readily exert executive control over drug-use urges post-treatment. Individuals who relapsed by day 30 had fewer years of education and reported a longer cigarette smoking history, but were otherwise similar to Nonrelapse individuals in demographic, clinical, treatment and neurocognitive characteristics measured here. Importantly, relapse-related differences in ECN connectivity were not related to years of education or years smoking.

Communication between the prefrontal cortices is supported by white matter tracts that travel through the anterior portion of the corpus collosum (Hofer and Frahm 2006). Reduced volume and 
compromised structural integrity of the corpus collosum have been associated with reduced frontal interhemispheric connectivity (Hinkley et al. 2012) as well as deficits in executive control (Meguro et al. 2003). Interhemispheric dorsolateral prefrontal cortical (dlPFC) connectivity has also been shown to increase with cognitive load (Fishburn et al. 2014), suggesting that as demand on frontal executive processes increases, so does the importance of interhemispheric coordination. In the present study, interhemispheric connectivity in prefrontal components of the ECN was not only associated with relapse risk, but also with perseverative responding on the WCST, a measure of cognitive flexibility known to be supported by the dlPFC (Demakis 2003).

Previous studies have observed reduced structural integrity within pathways that support frontal interhemispheric connectivity among CD individuals (e.g., Lim et al., 2008; Romero et al., 2010), as well as deficits in ECN interhemispheric resting connectivity and concomitant attentional lapses (Kelly et al. 2011). By contrast, effects in the present study do not suggest deficits in ECN interhemispheric connectivity as neither $\mathrm{CD}$ group evidenced reduced connectivity relative to $\mathrm{HC}$ individuals. Rather, our results suggest a sustained elevation in connectivity among individuals protected from early relapse.

If enhanced ECN connectivity is protective, an important follow-up question is can we manipulate ECN dynamics to modify relapse risk? Recent studies have shown that noninvasive stimulation of the left dIPFC via transcranial direct-current stimulation (tDCS), a method of delivering constant, low current to the cortical surface, produces an increase in dlPFC interhemispheric rsFC (Park et al. 2013) as well as improvements in executive function (e.g., Dockery et al., 2009). It may also be possible to promote interhemispheric connectivity simply through the delivery of psychosocial interventions (Penadés et al. 2013). 
Nonrelapse individuals also evidenced enhanced connectivity between the left ECN and dACC, a core component of the SN (Menon and Uddin 2010). The SN acts to detect the salience of new events and engage processing resources (e.g., ECN) required to respond adaptively (Menon and Uddin 2010). Thus where attention is externally oriented and executive control is required, increased co-activation and coupling between the SN and ECN is observed (Menon and Uddin 2010). Likewise, conditions characterized by deficits in cognitive function, such as nicotine withdrawal (Lerman et al. 2014) or mild cognitive impairment (Wu et al. 2014) are accompanied by a reduction in SN-ECN connectivity. Increased coupling between the left dIPFC (a component of the left ECN) and dACC may also enhance emotion regulation (Comte et al. 2014). Thus elevated left ECN to dACC coupling in Nonrelapse participants may provide enhanced capacity to engage the ECN when it is needed, such as when faced with opportunities to use cocaine or down regulate stress or craving.

Reduced risk of early relapse was also conferred by enhanced pre-discharge connectivity between the right ECN and left MTG. A part of the left ECN, the MTG is implicated in the storage of lexical representations (Jefferies 2013) and putatively provides meaning to our ongoing experiences and memories in order to guide goal-directed behavior (Jefferies 2013). A recent meta-analysis identified the left MTG as a key component in the neural network underlying the cognitive regulation of emotional responding (Kohn et al. 2014). Thus enhanced connectivity between the right ECN and left MTG may reflect a capacity to more readily engage this network in the face of stress or drug-related cues. Indeed, an earlier study found that cue exposure and training designed to reduce the salience of cocaine-related cues was accompanied by reduced left MTG activation to cocaine-cues (Prisciandaro et al. 2013), suggesting that as the salience of cues dissipates, the need to recruit the left MTG also declines. 
Finally, despite consistent evidence of DMN involvement in drug craving (for review see Sutherland et al., 2012), we found no relationship between resting DMN connectivity and relapse risk. This may reflect the state of individuals at the time resting scans were conducted. Prior to scan sessions, participants were abstinent for at least 14 days while completing treatment within a residential inpatient setting, away from drug-associated cues and environmental stressors. In such settings, cocaine-dependent individuals may experience little to no cocaine craving, even when at high risk of relapse post-discharge (Weiss, Griffin, and Hufford 2009). In the present study, cocaine-craving was generally low with $38 \%^{1}$ of CD individuals reported no cocaine craving at all. Self-reported craving was also unrelated to relapse risk. If the DMN participates in processing craving, it is possible that simply probing resting connectivity within this network at a time when craving is low may not prove sensitive to subsequent relapse risk. Of course, this does not rule out a role for the DMN in recidivism, and future studies that challenge the individual through the presentation of drug cues or acute abstinence from drugs of abuse may provide further insight into the contribution of this network to cocaine use relapse following treatment.

Some limitations of the current study should be noted. Firstly, we re-iterate that previous studies reported by our group have examined resting state connectivity in the present sample (Adinoff et al. 2015; McHugh et al. 2014; McHugh et al. 2013). To address concern of common variance contributing to effects across studies, we regressed mean connectivity within relapse-related pathways in the present study with those reported in our previous studies. No relationship between present and past effects was observed (regression coefficients ranged from -.182 to .177, ps >.153). This speaks to our earlier point that relapse is a multifaceted construct likely involving a myriad of distinct neural circuits. While we strongly encourage other investigators to obtain datasets to explore these questions further, our relatively

\footnotetext{
${ }^{1} 38 \%$ reflects the proportion of people within our CD cohort who scored 10 (the minimum score) on the CCQ. A score of 10 means that an individual did not endorse any craving-related symptoms.
} 
unique and meticulously collected data set offers an important opportunity to broaden our understanding of the neural circuits driving relapse in human cocaine-dependent individuals.

Due to high rates of relapse and general attrition, sample sizes for follow-up scans in the present sample were relatively small and underpowered. Our follow-up effects should thus be considered preliminary and null findings considered with caution. Neurocognitive and clinical assessments were also conducted on a separate occasion to the resting scan in the present study. This added state-dependent variance in both behavior and resting connectivity may have undermined our capacity to observe relationships between the two. Additionally, the absence of contemporaneous measures of craving, drug cue reactivity and withdrawal symptoms (although these would presume to have mostly resolved at 14 days abstinence) at the time of scanning also limited our ability to draw conclusions regarding the relationship between network connectivity, these state-dependent factors and relapse risk., Due to the lack of a pre-treatment pre-discharge measure of resting connectivity, it is also unknown whether enhanced connectivity in Nonrelapse individuals reflected a response to treatment or a pre-existing protective characteristic among these individuals. However, the primary purpose of the present study was to determine whether a snapshot of brain function immediately prior to treatment discharge could hold utility in predicting who is at risk of relapse, and ultimately who might require additional, more intensive treatment and relapse prevention efforts prior to discharge.

It is also important to note that participants in the present study were at least 2 weeks abstinent at the time of the pre-discharge scan, mitigating residual cocaine effects that might have been observed if the scan was obtained prior to treatment onset. Pre-treatment scans thus suffer the inherent limitation of being unable to distinguish residual cocaine effects and acute withdrawal effects from trait variance in network connectivity. Perhaps, future studies which take several snapshots of brain function across the course of 
treatment could help disentangle cocaine, withdrawal and treatment effects from pre-existing trait variance in network connectivity. Finally, future studies employing task-based fMRI paradigms could provide further insight into the functional mechanisms reflected in connectivity within and between SN, ECN and DMN and their relationship to relapse risk. Despite these limitations, the present study presents a promising new direction in characterizing and measuring relapse risk, as well as yielding a potential target for future interventions designed to reduce such risk. Future studies are needed to replicate the present findings, and subsequently, to manipulate connectivity within the ECN to directly test the causal role of this network in relapse to cocaine use following treatment.

\section{Acknowledgments}

This research was supported by the Intramural Research Program of the National Institute on Drug Abuse, NIDA grant DA023203 (BA) and ULT1R000451 (National Institutes of Health CTSA/UTSTAR). Drs McHugh, Gu, Yang, Adinoff and Stein report no biomedical financial interests or potential conflicts of interest.

\section{Author contributions}

BA, MM, and ES were responsible for the study concept and design. BA contributed to the acquisition of data. MM performed the data processing and analysis. HG and YY assisted with data analysis and interpretation of findings. MM drafted the manuscript. All authors provided critical revision

of the manuscript for important intellectual content and critically reviewed and approved the final version for publication. 


\section{References}

Adinoff B, Gu H, Merrick C, McHugh M, Jeon-Slaughter H, Lu H, Yang Y, Stein EA (2015) Basal Hippocampal Activity and Its Functional Connectivity Predicts Cocaine Relapse. Biol Psychiatry 78:496-504.

American Psychiatric A (1994) Diagnostic Stastistical Manual of Mental Disorders (4th ed). Author: Washington DC.

Biswal B, Yetkin FZ, Haughton VM, Hyde JS (1995) Functional Connectivity in the Motor Cortex of Resting. Magn Reson Med 34:537-541.

Buckner RL, Andrews-Hanna JR, Schacter DL (2008) The brain's default network: anatomy, function, and relevance to disease. Ann N Y Acad Sci 1124:1-38.

Chen BT, Yau H-J, Hatch C, Kusumoto-Yoshida I, Cho SL, Hopf FW, Bonci A (2013) Rescuing cocaineinduced prefrontal cortex hypoactivity prevents compulsive cocaine seeking. Nature 496:359362.

Comte M, Schön D, Coull JT, Reynaud E, Khalfa S, Belzeaux R, Ibrahim EC, Guedj E, Blin O, Weinberger DR, Fakra E (2014) Dissociating Bottom-Up and Top-Down Mechanisms in the Cortico-Limbic System during Emotion Processing. Cereb Cortex.

Connor CK, Staff MHS (2004) Conners' Continuous Performance Test II (CPT II): Version 5 for Windows Technical Guide and Software Manual. Multi-Health Systems Inc.: North Tonawanda, NY.

Cox RW (1996) AFNI: software for analysis and visualization of functional magnetic resonance neuroimages. Comput Biomed Res 29:162-173. 
Demakis GJ (2003) A meta-analytic review of the sensitivity of the Wisconsin Card Sorting Test to frontal and lateralized frontal brain damage. Neuropsychology 17:255-264.

Dockery CA, Hueckel-Weng R, Birbaumer N, Plewnia C (2009) Enhancement of planning ability by transcranial direct current stimulation. J Neurosci 29:7271-7277.

Dutra L, Ph D, Stathopoulou G, Basden SL, Leyro TM, Powers MB, Otto MW (2008) A Meta-Analytic

= Review of Psychosocial Interventions for Substance Use Disorders. Am J Psychiatry 165:179187.

Erb S (2009) Evaluation of the relationship between anxiety during withdrawal and stress-induced reinstatement of cocaine seeking. Prog Neuropsychopharmacol Biol Psychiatry 34:798-807.

Fedota JR, Stein EA (2015) Resting-state functional connectivity and nicotine addiction: prospects for biomarker development. Ann N Y Acad Sci 1349:64-82.

Filippini N, MacIntosh BJ, Hough MG, Goodwin GM, Frisoni GB, Smith SM, Matthews PM, Beckmann CF, Mackay CE (2009) Distinct patterns of brain activity in young carriers of the APOE-epsilon4 allele. Proc Natl Acad Sci U S A 106:7209-7214.

Fishburn FA, Norr ME, Medvedev AV, Vaidya CJ (2014) Sensitivity of fNIRS to cognitive state and load. Front Hum Neurosci 8:76-76.

Geng X, Christensen GE, Gu H, Ross TJ, Yang Y (2009) Implicit reference-based group-wise image registration and its application to structural and functional MRI. Neuroimage 47:1341-1351.

Goldstein RZ, Volkow ND (2002) Drug addiction and its underlying neurobiological basis: neuroimaging -evidence for the involvement of the frontal cortex. Am J Psychiatry 159:1642-1652.

Greicius M (2008) Resting-state functional connectivity in neuropsychiatric disorders. Curr Opin Neurol 21:424-430. 
Heaton RK, et al. (1993) Wisconsin Card Sorting Test Manual: Revised and Expanded. Psychological Assessment Resources: Odessa, FLa.

Hinkley LBN, Marco EJ, Findlay AM, Honma S, Jeremy RJ, Strominger Z, Bukshpun P, Wakahiro M, Brown WS, Paul LK, Barkovich AJ, Mukherjee P, Nagarajan SS, Sherr EH (2012) The role of corpus callosum development in functional connectivity and cognitive processing. PLoS One $=7: \mathrm{e} 39804-\mathrm{e} 39804$.

Hofer S, Frahm J (2006) Topography of the human corpus callosum revisited--comprehensive fiber tractography using diffusion tensor magnetic resonance imaging. Neuroimage 32:989-994.

Jefferies E (2013) The neural basis of semantic cognition: converging evidence from neuropsychology, neuroimaging and TMS. Cortex 49:611-625.

Kalivas PW (2008) Addiction as a pathology in prefrontal cortical regulation of corticostriatal habit circuitry. Neurotox Res 14:185-189.

Kass R, Raftery, A. (1995) Bayes factors. Journal of the American Statistical Association 90:773-795.

Kelly C, Zuo X-N, Gotimer K, Cox CL, Lynch L, Brock D, Imperati D, Garavan H, Rotrosen J, Castellanos FX, Milham MP (2011) Reduced interhemispheric resting state functional connectivity in cocaine addiction. Biol Psychiatry 69:684-692.

Kohn N, Eickhoff SB, Scheller M, Laird aR, Fox PT, Habel U (2014) Neural network of cognitive emotion regulation--an ALE meta-analysis and MACM analysis. Neuroimage 87:345-355.

Lim KO, Wozniak JR, Mueller BA, Franc DT, Specker SM, Rodriguez CP, Silverman AB, Rotrosen JP (2008) Brain macrostructural and microstructural abnormalities in cocaine dependence. Drug Alcohol Depend 92:164-172.

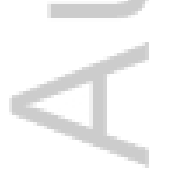


McHugh MJ, Demers CH, Braud J, Briggs R, Adinoff B, Stein EA (2013) Striatal-insula circuits in cocaine addiction: implications for impulsivity and relapse risk. Am J Drug Alcohol Abuse 39:424-432.

McHugh MJ, Demers CH, Salmeron BJ, Devous MD, Stein EA, Adinoff B (2014) Cortico-amygdala coupling as a marker of early relapse risk in cocaine-addicted individuals. Front Psychiatry 5:16$=16$.

Meguro K, Constans J-M, Shimada M, Yamaguchi S, Ishizaki J, Ishii H, Yamadori A, Sekita Y (2003) Corpus callosum atrophy, white matter lesions, and frontal executive dysfunction in normal aging and Alzheimer's disease. A community-based study: the Tajiri Project. Int Psychogeriatr 15:9-25. Menon V (2011) Large-scale brain networks and psychopathology: a unifying triple network model.

Trends Cogn Sci 15:483-506.

Naqvi NH, Bechara A (2009) The hidden island of addiction: the insula. Trends Neurosci 32:56-67.

Park C-H, Chang WH, Park J-Y, Shin Y-I, Kim ST, Kim Y-H (2013) Transcranial direct current stimulation increases resting state interhemispheric connectivity. Neurosci Lett 539:7-10.

Penadés R, Pujol N, Catalán R, Massana G, Rametti G, García-Rizo C, Bargalló N, Gastó C, Bernardo M, Junqué C (2013) Brain effects of cognitive remediation therapy in schizophrenia: a structural and functional neuroimaging study. Biol Psychiatry 73:1015-1023.

Poling J, Kosten TR, Sofuoglu M (2007) Treatment outcome predictors for cocaine dependence. Am J Drug Alcohol Abuse 33:191-206.

Prisciandaro JJ, Myrick H, Henderson S, McRae-Clark AL, Santa Ana EJ, Saladin ME, Brady KT (2013) Impact of DCS-facilitated cue exposure therapy on brain activation to cocaine cues in cocaine dependence. Drug Alcohol Depend 132:195-201. 
Romero MJ, Asensio S, Palau C, Sanchez A, Romero FJ (2010) Cocaine addiction: diffusion tensor imaging study of the inferior frontal and anterior cingulate white matter. Psychiatry Res 181:5763.

Rounder, IN, Speckman, PL, Sun, D, Morey, RD, Iverson, G (2009) Bayesian t-tests for accepting and rejecting the null hypothesis. Psychonomic Bulletin \& Review 16:225-237.

See RE (2005) Neural substrates of cocaine-cue associations that trigger relapse. Eur J Pharmacol 526:140-146.

Shirer WR, Ryali S, Rykhlevskaia E, Menon V, Greicius MD (2012) Decoding subject-driven cognitive states with whole-brain connectivity patterns. Cereb Cortex 22:158-165.

Stewart J (2008) Review. Psychological and neural mechanisms of relapse. Philos Trans R Soc Lond B Biol Sci 363:3147-3158.

Sutherland MT, McHugh MJ, Pariyadath V, Stein Ea (2012) Resting state functional connectivity in addiction: Lessons learned and a road ahead. Neuroimage 62:2281-95

Tiffany ST, Singleton E, Haertzen Ca, Henningfield JE (1993) The development of a cocaine craving questionnaire. Drug Alcohol Depend 34:19-28.

Weiss RD, Griffin ML, Hufford C (1995) Craving in Hospitalized Cocaine Abusers as a Predictor of Outcome. Am J Drug Alcohol Abuse 21:289-301.

Woicik Pa, Urban C, Alia-Klein N, Henry A, Maloney T, Telang F, Wang G-J, Volkow ND, Goldstein RZ (2011) A pattern of perseveration in cocaine addiction may reveal neurocognitive processes implicit in the Wisconsin Card Sorting Test. Neuropsychologia 49:1660-1669. 


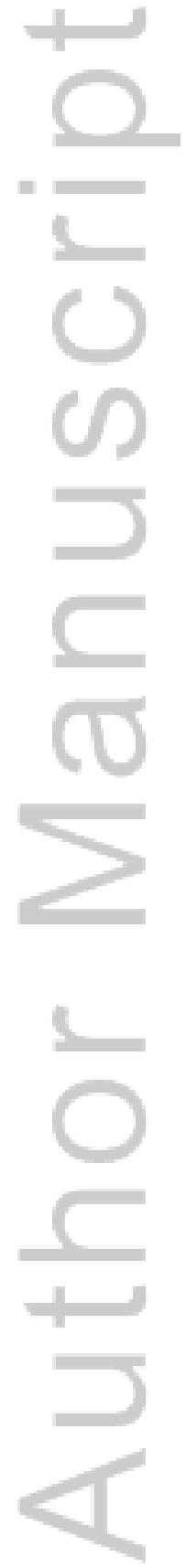

This article is protected by copyright. All rights reserved. 
Table 1. Demographic, drug use, treatment, and behavioral characteristics.

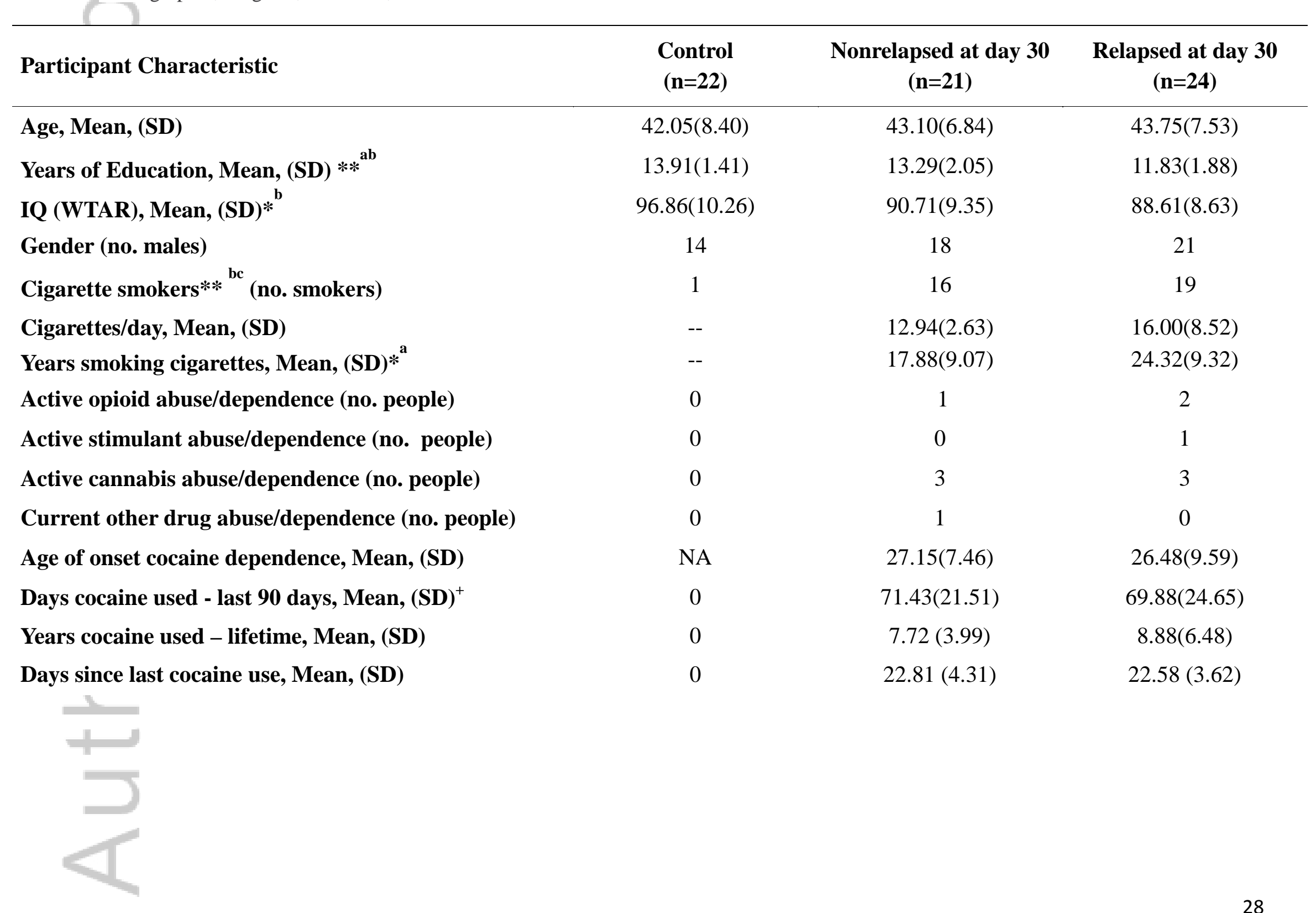


Amount spent on cocaine - last 90 days, Mean, (SD)

0

Treatment centre (no. in $\mathbf{A}, \mathbf{B}, \mathbf{C}) \hat{}$

Treatment duration in days, Mean, (SD))

Craving - CCQ-Brief, Mean, (SD)

WCST - perseverative responses, Mean, $(\mathrm{SD})^{\# * \mathrm{~b}}$

CPT - commissions, Mean, (SD)

CPT - omissions, Mean, (SD)

CPT - Hit reaction time (ms), Mean, (SD)

CPT - Hit reaction time standard error, Mean, (SD)

Index of head motion during resting scan, Mean, (SD)

Percentage $(\%)$ censored points, Mean (SD)

Days to relapse post-discharge, Mean (SD) $* * *^{\mathrm{a}}$
NA

NA

NA

$14.43(13.74)$

1.62(2.85)

9.62(5.67)

398.35(62.06)

4.80(1.39)

$.10(.03)$

$.34(.77)$

NA
$\$ 8075.05(6296.51)$

$3,16,2$

24.74(8.08)

19.48(10.73)

17.21(9.71)

1.95 (2.63)

10.21(6.73)

398.29(65.13)

5.39(1.83)

$.10(.02)$

$.43(.66)$

110.57 (55.58)
$\$ 5910.67(5484.34)$

$7,14,3$

26.19(14.04)

18.39(13.15)

24.79(16.9)

$3.13(3.62)$

12.56(7.73)

$384.86(57.37)$

5.11(1.56)

$.11(.05)$

$.69(1.32)$

$8.13(5.65)$

$* \mathrm{p}<.05 * * \mathrm{p}<.01 * * * \mathrm{p}<.001$ for group main effects. a. Difference between Relapse and Nonrelapse; b. Difference between relapse and controls; c. Difference between Nonrelapse and Controls.

${ }^{\wedge}$ Treatment centers based in Dallas, TX, A = Veteran's Administration Medical Center; B = Homeward Bound Inc.; C= Nexus Recovery Center.

${ }^{+}$Last 90 days refers to period prior to admission for this treatment episode.

\# Values reflect raw perseverative responses, which were natural $\log (\ln )$ transformed prior to conducting between-group ANOVA.

Percentage of TRs censored due to excessive motion.

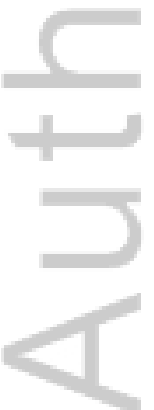

This article is protected by copyright. All rights reserved. 
Note Cocaine-dependent participants had been abstinent from all drugs of abuse for a minimum of 17 days at the day of scanning. Also, a portion of the findings presenting in Table 1 have been reported in two previous studies (McHugh et al. 2014; McHugh et al. 2013).

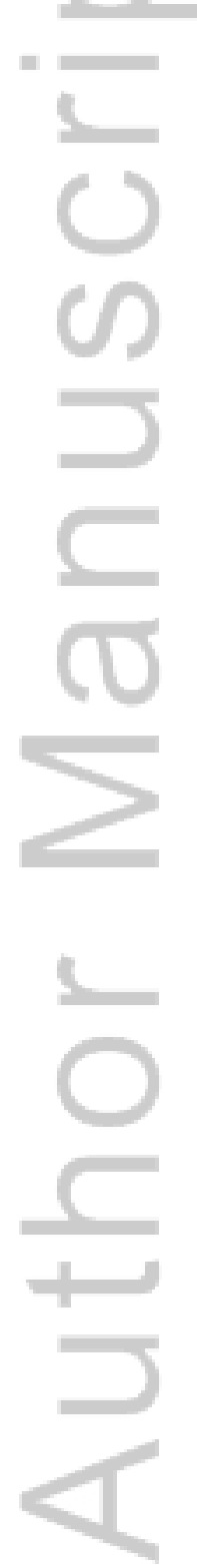

This article is protected by copyright. All rights reserved. 
Table 2. Summary of significant activation arising from voxelwise Relapse vs Nonrelapse contrasts.

$(2)$

Resting connectivity contrast

Relapse vs Nonrelapse

(Network to cluster)

Cluster Size

\section{Peak}

Differences

F value

(3x3x3mm voxels)

F value
21.7

18.73

21.22

32.52

Right dorsal anterior cingulate cortex

251
185
52
41

41

50

20.45

$-49.75$

25.25
28.25
-31.75
13.25

19.25

35.25

5.25

$-30.75$

$-61.75$

$-30.75$

Right ECN

Left middle temporal gyrus

50

Note. Effects were reported based on a

voxelwise threshold $p$

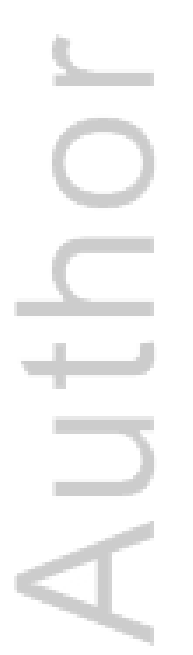

This article is protected by copyright. All rights reserved. 
Table 3. Summary of significant activation arising from voxelwise regression analysis of perseverative responding on the Wisconsin Card Sorting (

Task (WCST) against resting state connectivity for left and right Executive Control Networks (ECNs).

a

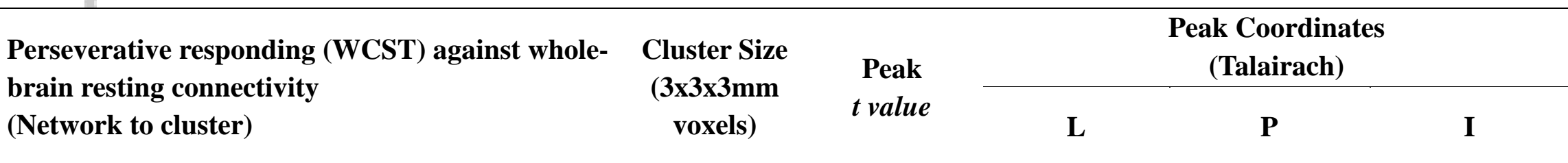

Left ECN

Right middle frontal gyrus (right ECN)

48

$-4.74$

49.25

40.25

5.25

Right ECN

$\begin{array}{llllll}\text { Right supramarginal gyrus (right ECN) } & 56 & -3.12 & 61.25 & -52.75 & 26.25 \\ \text { Right middle temporal gyrus (right ECN) } & 43 & -3.74 & 49.25 & -28.75 & -0.75\end{array}$

Note. Effects were reported based on a voxelwise threshold $p_{\text {uncorrected }}=.005$, and clusterwise threshold at $p_{\text {corrected }}=.05$. Reported for all individuals with valid WCST and pre-discharge resting state connectivity measures $(n=58)$.

This article is protected by copyright. All rights reserved. 


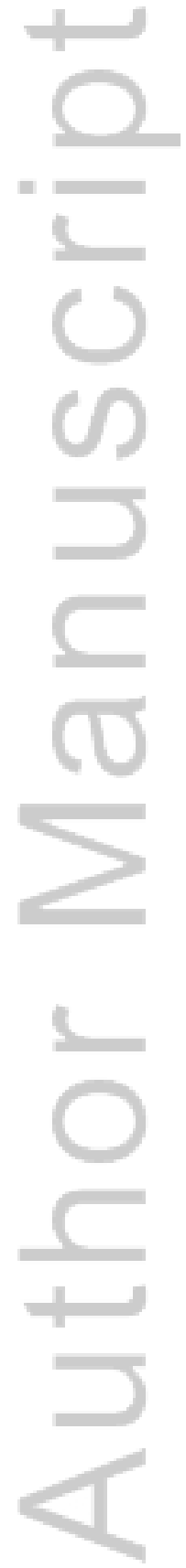

This article is protected by copyright. All rights reserved. 


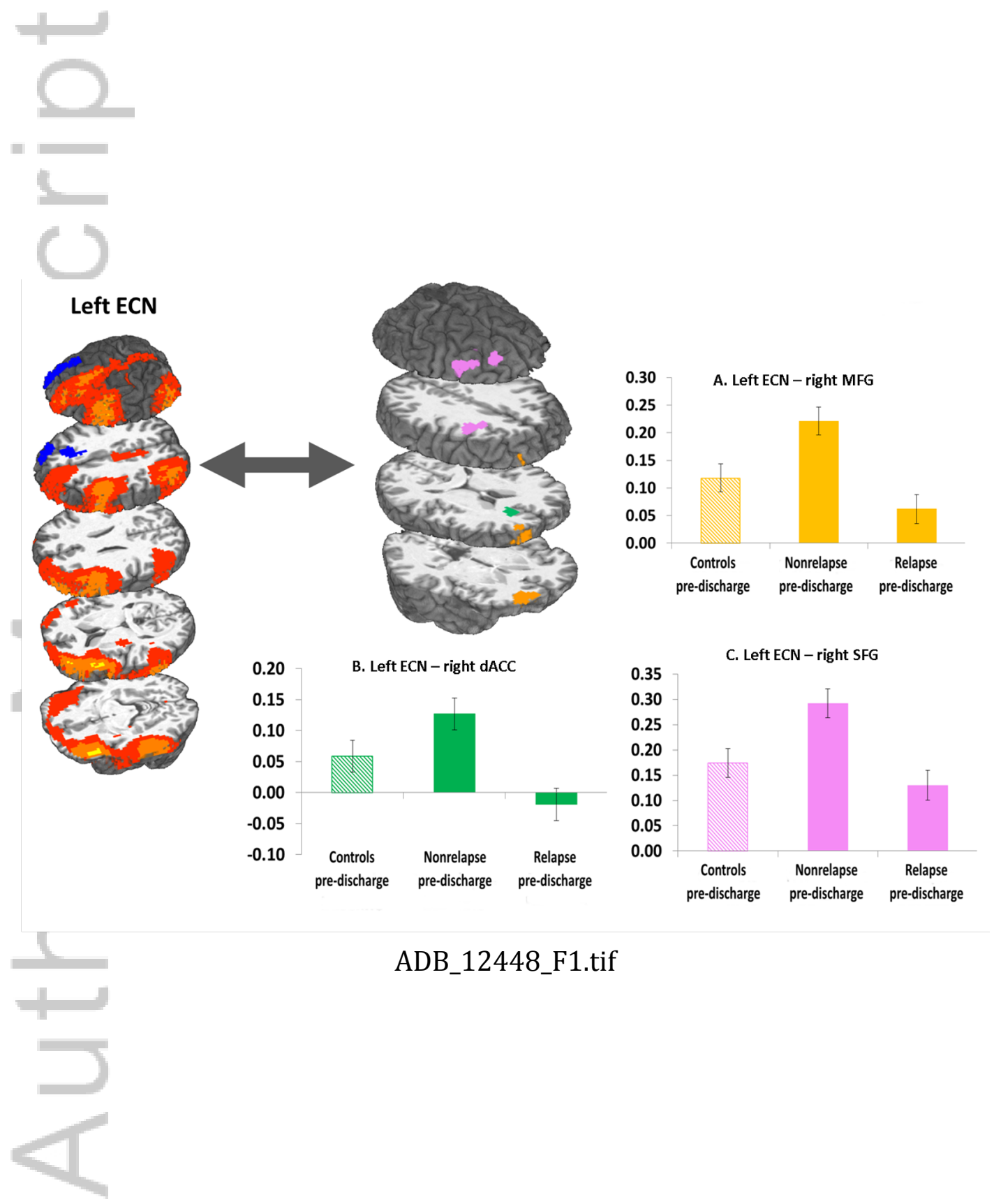

This article is protected by copyright. All rights reserved. 

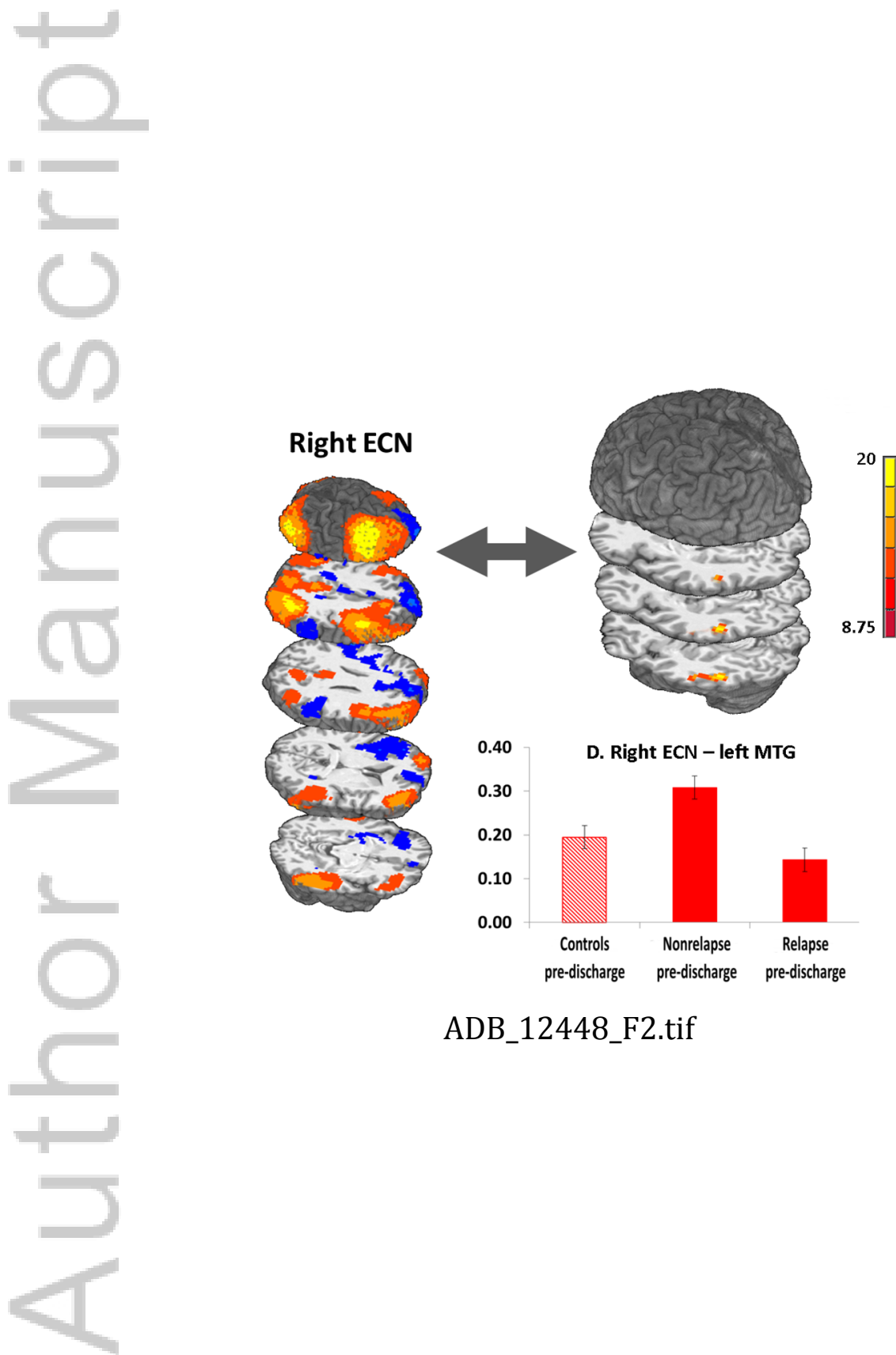

ADB_12448_F2.tif

This article is protected by copyright. All rights reserved. 

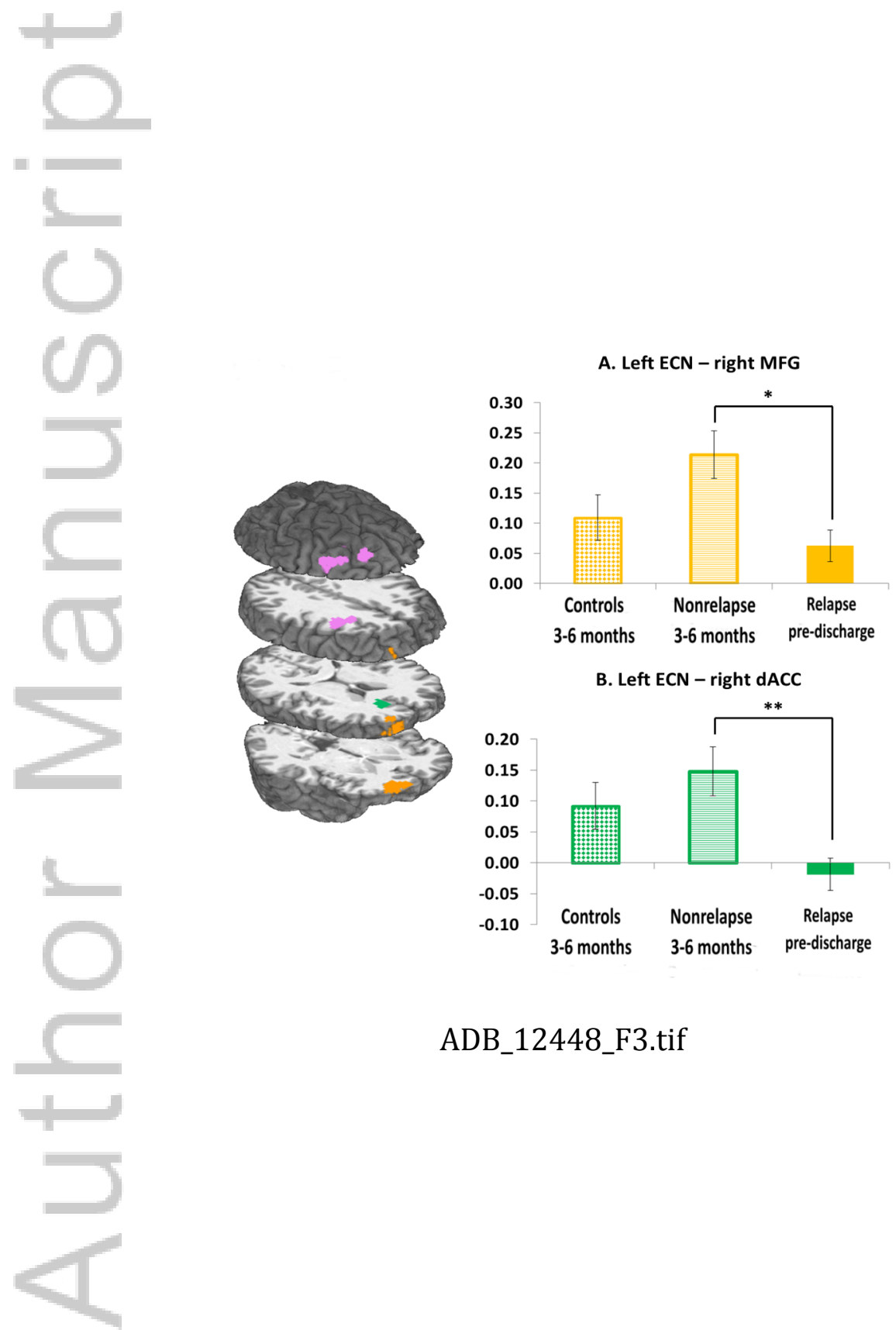

ADB_12448_F3.tif

This article is protected by copyright. All rights reserved. 

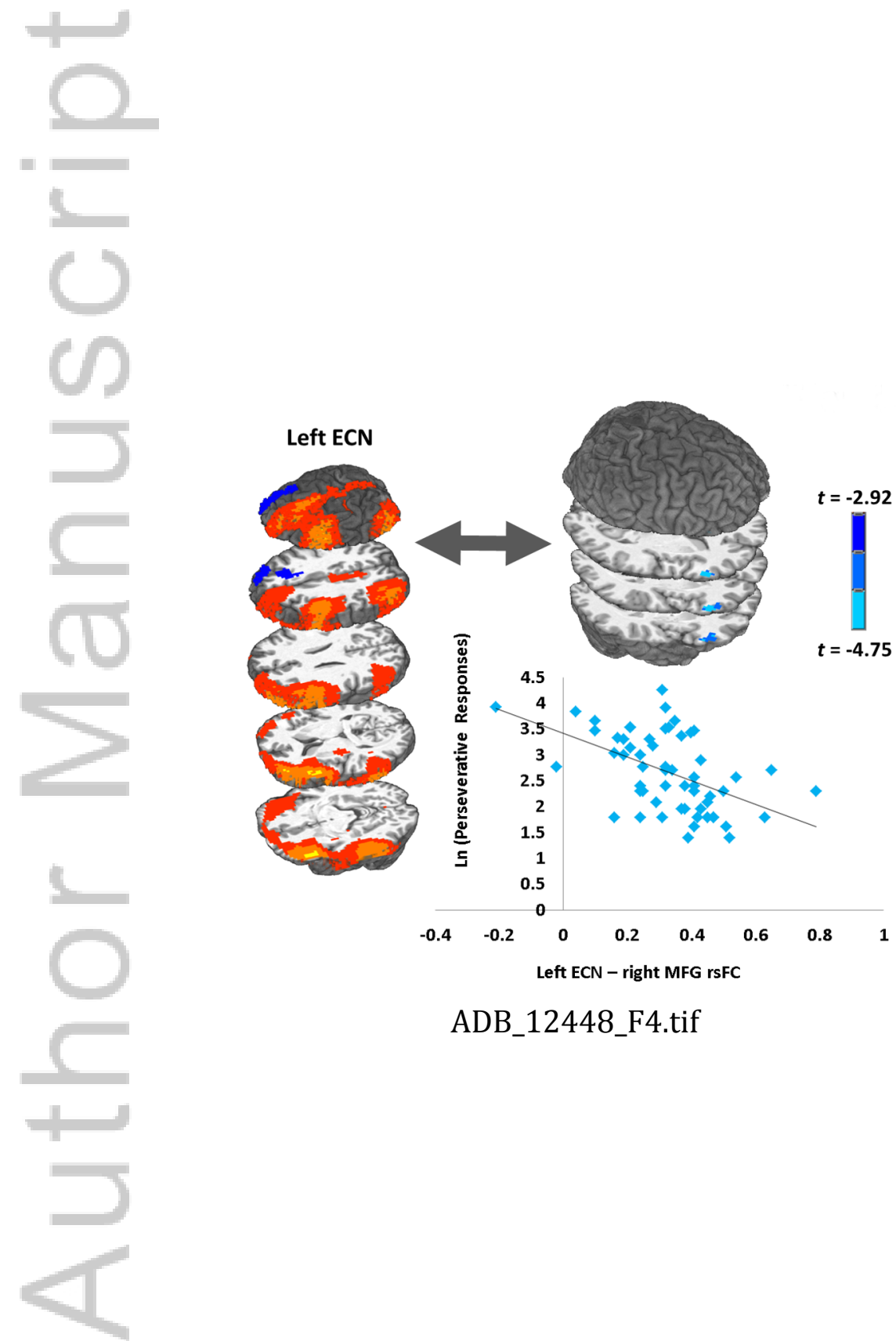

ADB_12448_F4.tif

This article is protected by copyright. All rights reserved. 


\section{FIGURE LEGENDS}

Figure 1. Spatial map and mean connectivity graphs displaying significantly elevated connectivity between the left ECN and (A) right middle frontal gyrus, MFG; (B) right dorsal anterior cingulate cortex, dACC; and (C) right superior frontal gyrus, SFG, among individuals who remained abstinent during the first 30 days post-treatment (Nonrelapse, $n=21$ ) relative to those who relapsed to cocaine use during this period (Relapse, $n=24)$. Control participants $(n=22)$ are displayed for comparison purposes, but did not significantly differ from either Relapse or Nonrelapse groups. Error Bars reflect SEM. Effects are reported at a voxelwise threshold of $p_{\text {uncorrected }}=.005$, and a corrected clusterwise threshold of $p_{\text {corrected }}=$ .05 .

Figure 2. Spatial map and mean connectivity graphs displaying significantly elevated connectivity between the right ECN and (A) left middle temporal gyrus, MTG among individuals who remained abstinent during the first 30 days post-treatment (Nonrelapse, $n=21$ ) relative to those who relapsed to cocaine use during this period (Relapse, $n=24)$. Control participants $(n=22)$ are displayed for comparison purposes, but did not significantly differ from either Relapse or Nonrelapse groups. Error Bars reflect SEM. Effects are reported at a voxelwise threshold of $p_{\text {uncorrected }}=.005$, and a corrected clusterwise threshold of $p_{\text {corrected }}=.05$.

Figure 3. Mean connectivity was extracted from clusters where significant Relapse vs Nonrelapse group differences were present pre-discharge (spatial map in left panel displays clusters). Extracted mean connectivity between (A) left ECN and right middle frontal gyrus (MFG); and between (B) left ECN and right dorsal anterior cingulate cortex $(\mathrm{dACC})$ is displayed for Control $(n=10)$ and Nonrelapse individuals $(n=9)$ at $3-6$ months follow-up (right panel). Pre-discharge connectivity in both clusters is also illustrated for Relapse individuals $(n=24)$. Posthoc contrasts indicated that mean connectivity in both clusters remained significantly elevated among Nonrelapse individuals at 3-6 months post-discharge 
relative to pre-discharge mean connectivity of Relapse individuals. Error bars reflect SEM. * $p<.05$, ** $p$ $<.01$.

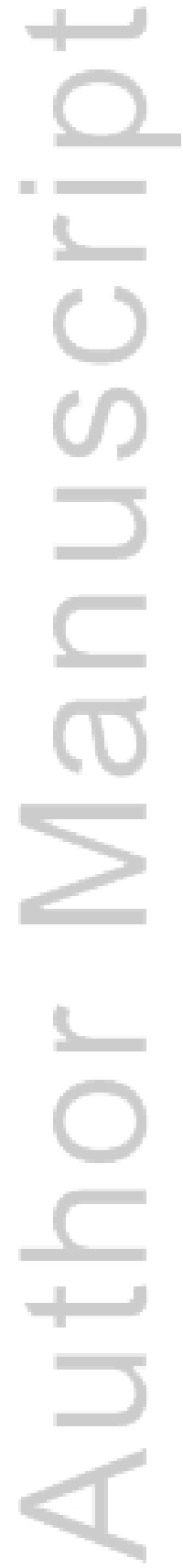

This article is protected by copyright. All rights reserved. 
Figure 4. Top right: Spatial map illustrating whole-brain connectivity between the left ECN and right middle frontal gyrus (MFG) as a function of number of perseverative responses on the Wisconsin Card Sorting Task (WCST) across all study participants with WCST data $(n=59)$ Bottom right: Scatterplot of mean connectivity extracted from above cluster against perseverative responses on the WCST. Ln = natural $\log$ transform. Effects are reported at a voxelwise threshold of $p_{\text {uncorrected }}=.005$, and a corrected clusterwise threshold of $p_{\text {corrected }}=.05$.

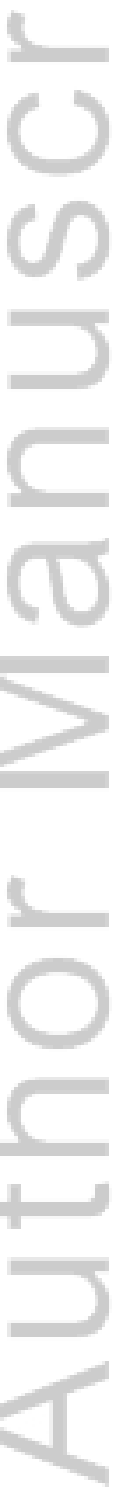

This article is protected by copyright. All rights reserved. 


\section{University Library}

\section{- M M I N E R VA A gateway to Melbourne's research publications}

Minerva Access is the Institutional Repository of The University of Melbourne

Author/s:

McHugh, MJ;Gu, H;Yang, Y;Adinoff, B;Stein, EA

Title:

Executive control network connectivity strength protects against relapse to cocaine use

Date:

2017-11-01

Citation:

McHugh, M. J., Gu, H., Yang, Y., Adinoff, B. \& Stein, E. A. (2017). Executive control network connectivity strength protects against relapse to cocaine use. ADDICTION BIOLOGY, 22 (6), pp.1790-1801. https://doi.org/10.1111/adb. 12448.

Persistent Link:

http://hdl.handle.net/11343/291700 